\title{
Linear Massive MIMO Uplink Detector Based On Joint Jacobi and Gauss-Seidel Methods
}

\author{
Mahmoud A. M. Albreem and Ayman A. El-Saleh \\ A'Sharqiyah University \\ Ibra, Oman \\ Email: mahmoud.albreem@asu.edu.om
}

\author{
Markku Juntti \\ University of Oulu \\ Oulu, Finland \\ Email: markku.juntti@oulu.fi
}

\begin{abstract}
In fifth generation (5G) cellular system, massive multiple-input multiple-output (MIMO) is utilized to improve the diversity gain, reliability, link robustness, latency, and power and spectral efficiencies. However, a large number of antennas requires sophisticated signal processing to detect data. Although the detection based on maximum likelihood (ML) obtains the best performance, it is not hardware friendly because of the exponential complexity. Therefore, several iterative methods are proposed to estimate the signal without computing the inverse of equalization matrix, and hence, minimize the complexity. The Jacobi (JA) and the Gauss-Seidel (GS) methods achieve a satisfactory performance. However, large iterations' number is in demand which produces a high computational complexity. This paper proposes a detector for massive MIMO uplink (UL) system based on the JA and GS methods. Proposed detector obtains a balance between the performance and the complexity. In this research, initialization is performed based on the JA method. After-that, the estimation is performed based on the GS method. Numerical results show that the proposed JAGS detector outperforms the GS and the JA based detector. Moreover, proposed JA-GS based detector requires few iterations to obtain the target performance and hence, a considerable reduction in computational complexity is achieved.
\end{abstract}

Index Terms-Massive MIMO, MMSE, Neumann series, GausSeidel, Jacobi

\section{INTRODUCTION}

The strong momentum for fifth generation (5G) communication system continues. In 2019, the number of mobile subscriptions have exceeded 7.9 billion. Over 10 million and 1.9 billion $5 \mathrm{G}$ subscriptions are expected worldwide by the end of 2019 and 2024, respectively [1]. High quality of service (QoS) is a target for mobile companies. Therefore, several key technologies are employed in 5G systems. The device to device (D2D) communication can boost the accuracy of communication between transceivers by reducing the latency. In the ultra dense networks (UDNs), a dense small cell deployment is involved, in areas with extremely high traffic intensity. The spectrum sharing (SS) role is to avert low spectrum utilization efficiency. In order to meet larger spectrum channels, and hence, multi Gigabits per second data rates, millimeter wave (mmWave) uses the spectrum from $30 \mathrm{GHz}$ to $300 \mathrm{GHz}$ [2]. It requires highly directive beams that can maximize energy transfer to targeted user [3], [4]. The visible light communication (VLC) is a fast speed technology which provides unregulated bandwidth and high data rate communication for indoor environment [5]. The internet of things (IoT) connects billions of devices, machines, and gadgets. Therefore, the base station (BS) is equipped with a massive number of antennas to serve a huge number of devices envisioned by the IoT [6], [7]. In massive massive multiple-input multiple-output (MIMO), large number of antennas are employed at the BS to turn many user terminals. Massive MIMO enhances the link robustness, latency, and power and spectrum efficiencies. However, large number of antennas needs sophisticated signal processing techniques to equalize the signal [8]. Optimal detection in smallscale MIMO technology needs an exhaustive search over all possible transmit symbol vectors. Therefore, the complexity is too high. The case is coming worse when a large-scale MIMO system is applied where a large number of antenna elements are used at both transmitter and receiver sides [9]. However, the maximum likelihood (ML) obtains the best performance but it is prohibited in realization due to high complexity. In literature, intensive research has been conducted to propose efficient low complexity detectors where a survey is presented in [10]. For instance, detectors based on the sphere decoding (SD) [11], the successive interference cancellation (SIC) , sparsity based algorithms, deep learning and neural network algorithms for detection purposes, and lattice reductionaided (LRA) techniques [12], [13]. Although they achieve a satisfactory performance, their complexity is still high. A detector based on linear minimum mean square error (MMSE) is an alternative solution but it computes the matrix inversion which increases the complexity. Several iterative methods are presented to approximate or avert the matrix inversion such as the successive over relaxation (SOR), Gauss-Seidel (GS) , Newton iterations (NI), Jacobi (JA), conjugate gradient (CG), Neumann series (NS), and Richardson (RI)methods. The complexity of iterative methods is greatly affected by the number of iterations. However, the GS and JA methods obtains a satisfactory performance with a low complexity if the base station (BS)-to-user-antenna-ratio (BUAR) is small.

In this paper, the JA and GS methods are exploited to propose a low complexity detector for massive MIMO UL system. It is initialized by the JA method while estimation of the signal is performed using the GS method. Section II presents the background and the system model. Section III presents the joint detector and the initialization technique. Section IV presents the complexity analysis and the impact of the proposed initialization on the complexity. Section V 
exhibits the result and the discussion. Section VI summarizes the paper.

\section{BACKGROUND}

\section{A. System Model}

In massive MIMO UL system, many user terminals $(K)$ are served by massive number of antennas $(N)$ at the BS where $N \gg K$. However, transmitted and received signals are presented as $\mathbf{x}=\left[x_{1}, x_{2}, \ldots ., x_{K}\right]^{T}$ and $\mathbf{y}=\left[y_{1}, y_{2}, \ldots ., y_{N}\right]^{T}$, respectively. The relationship among $\mathbf{x}, \mathbf{y}$, and $\mathbf{H}$ is

$$
\mathbf{y}=\mathbf{H x}+\mathbf{n},
$$

where $\mathbf{n}$ is the $N \times 1$ noise vector. The optimum ML detector exhaustively searches all possible solutions to estimate the signal as

$$
\hat{\mathbf{x}}=\arg \min _{x \in|C|^{K}}\left\|\mathbf{y}-\sqrt{\frac{S N R}{K}} \mathbf{H x}\right\|^{2},
$$

where $\hat{\mathbf{x}}, C$, and SNR, are the estimated signal, the modulation alphabet, and the signal-to-noise ratio, respectively. The complexity of ML algorithm is exponential and hence, it is prohibited in realization. Linear minimum mean square error (MMSE) method is a popular alternative solution to equalize the signal as

$$
\hat{\mathbf{x}}=\mathbf{A}^{-1} \mathbf{y}_{M F},
$$

and

$$
\mathbf{A}=\mathbf{G}+\sigma^{2} \mathbf{I}_{K}
$$

where $\mathbf{G}$ is the Gram matrix and $\mathbf{G}=\mathbf{H}^{H} \mathbf{H}, \sigma^{2}$, and $\mathbf{I}_{K}$ are the noise variance and the $K \times K$ identity matrix, respectively. The complexity of computing the $\mathbf{A}^{-1}$ is very high and the problem becomes more dominant when a large size of $(\mathbf{A})$ is utilized. Therefore, several iterative methods are proposed to approximate or avert the inverse of $\mathbf{A}$.

\section{B. Neumann series}

The Gram matrix (G) is decomposed into non-diagonal matrix (E) and a main diagonal matrix (D) where $\mathbf{G}=\mathbf{D}+\mathbf{E}$. In the NS method, $\left(\mathbf{A}^{-1}\right)$ can be written as

$$
\mathbf{A}^{-1}=\sum_{i=0}^{\infty}\left(\mathbf{I}-\mathbf{D}^{-1} \mathbf{E}\right)^{i} \mathbf{D}^{-1} .
$$

However, if the condition

$$
\lim _{i \rightarrow \infty}\left(\mathbf{I}-\mathbf{D}^{-1} \mathbf{E}\right)^{i}=0
$$

is satisfied, (5) converges to $\mathbf{A}^{-1}$. The approximate solution in (5) is refined iteratively. The NS based detector has high complexity in comparison with the GS, JA, SOR, and RI methods. It also sustains a significant loss in performance if the BUAR is high.

\section{Gauss-Seidel}

In the GS method, the signal can be estimated as

$$
\hat{\mathbf{x}}_{(n)}=(\mathbf{D}+\mathbf{L})^{-1}\left(\hat{\mathbf{y}}_{M F}-\mathbf{U} \hat{\mathbf{x}}_{(n-1)}\right), \quad n=1,2, \cdots,
$$

where $\hat{\mathbf{y}}_{M F}=\mathbf{H}^{H} \mathbf{y}, \mathbf{D}, \mathbf{L}$ and $\mathbf{U}$ are the diagonal entries, lower triangular matrix, and upper triangular matrix, respectively. The GS based detector obtains an acceptable performance even if the BUAR is high.

\section{Jacobi Method}

In comparison with the GS method, the JA method has a low convergence rate. The signal can be estimated as

$$
\hat{\mathbf{x}}_{(n)}=\mathbf{D}_{-1}\left(\hat{\mathbf{y}}_{M F}+(\mathbf{D}-\mathbf{A}) \hat{\mathbf{x}}^{(n-1)}\right) .
$$

The JA based detector can be implemented with ease, but it is not robust and not fast as the GS method.

\section{Proposed Method}

In this paper, the JA and GS methods are utilized to design a low complexity massive MIMO UL detector. Figure 1 presents a general block diagram of the proposed JA-GS based detector. The detector contains two stages, the initialization and the final estimation. The initial solution is computed based on the JA method as

$$
\hat{\mathbf{x}}_{(0)}=\mathbf{D}^{-1} \mathbf{y}_{M F} .
$$

Apply the first iteration in JA method as

$$
\hat{\mathbf{x}}_{(1)}=\mathbf{D}^{-1}\left(\hat{\mathbf{x}}_{M F}+(\mathbf{D}-\mathbf{A}) \hat{\mathbf{x}}_{(0)}\right) .
$$

After that, the initial solution is refined iteratively and the estimation of the signal is performed based on the GS method as shown in (7).

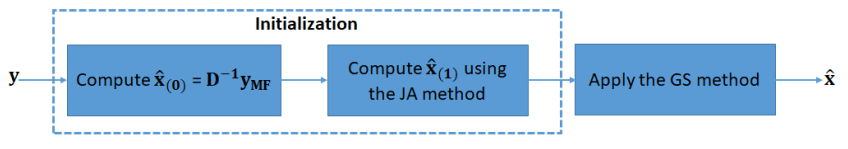

Figure 1. Block diagram of the proposed JA-GS based detector

Algorithm 1 presents proposed joint detector using the JA and GS methods.

\section{Complexity AnAlysis}

Multiplications and divisions are the most dominant operation in the computational complexity analysis. The GS method requires a $4 n K^{2}$ number of multiplications and the JA method requires a $n\left(4 K^{2}-2 K\right)$ number of multiplications. The proposed JA-GS detector requires a $4 K^{2}-2 K$ to initialize the detector and $4 n K^{2}$ to estimate the signal. Therefore, complexity of proposed JA-GS detector is expressed as $4 K^{2}(n+1)-2 K$. Proposed JA-GS needs a small number of iterations to attain the target performance and hence, less computational complexity is required. 

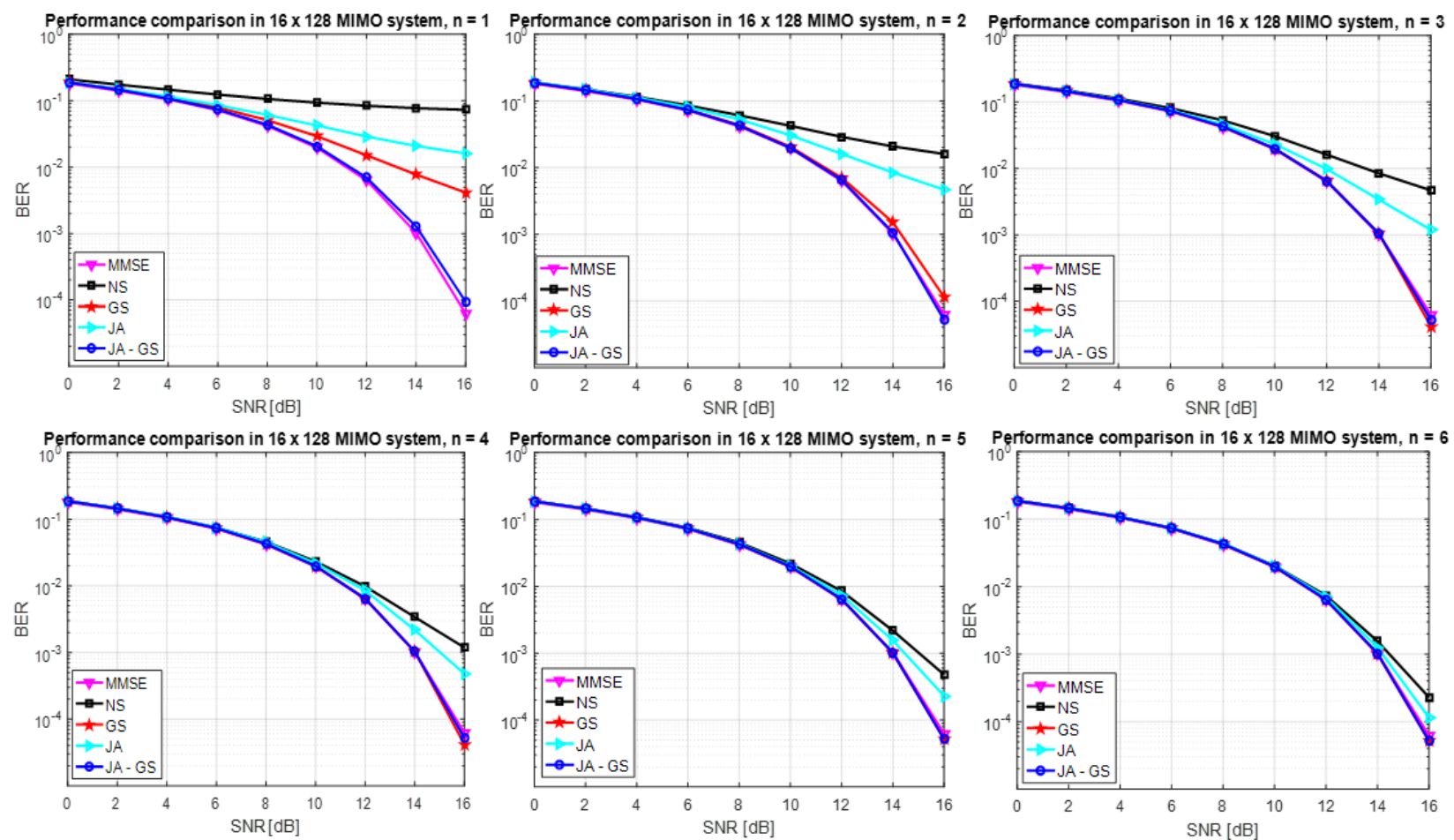

Figure 2. Performance of the NS, the GS, the JA and proposed JA-GS based detector in $16 \times 128$ MIMO system

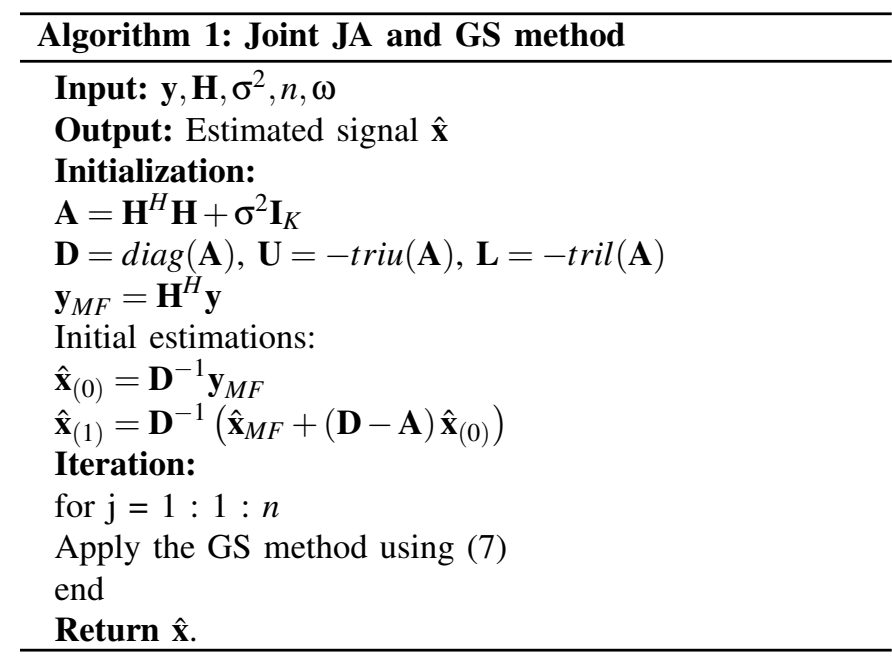

Table I

COMPLEXITY OF THE NS, GS, JA AND PROPOSED JA-GS METHODS.

\begin{tabular}{|l|l|}
\hline Method & Complexity \\
\hline GS & $4 n K^{2}$ \\
\hline JA & $n\left(4 K^{2}-2 K\right)$ \\
\hline NS & $(n-2) K^{3}+N K^{2}+N K$ \\
\hline Proposed JA-GS & $4 K^{2}(n+1)-2 K$ \\
\hline
\end{tabular}

\section{RESULTS}

The performance and the complexity of proposed JA-GS detector are presented. A comparison is conducted between performance-complexity profiles of proposed detector and the
GS, JA, and NS methods. Performance and complexity are presented in bit-error-rate (BER) and number of multiplications, respectively. Independent and identically distributed (i.i.d) Gaussian channels and $16 \times 128$ MIMO system are considered as well as the modulation scheme is 64QAM.

Figure 2 presents proposed proposed JA-GS method's performance. Proposed detector outperforms the GS, JA, and NS methods. In order to obtain a $\mathrm{BER}=10^{-3}$, proposed detector, GS, JA, and NS achieve the target BER at SNR $=14 \mathrm{~dB}$ and $n=1, \mathrm{SNR}=14.5 \mathrm{~dB}, n=2, \mathrm{SNR}=16 \mathrm{~dB}, n=3$, and $\mathrm{SNR}$ $=16 \mathrm{~dB}, n=4$, respectively.

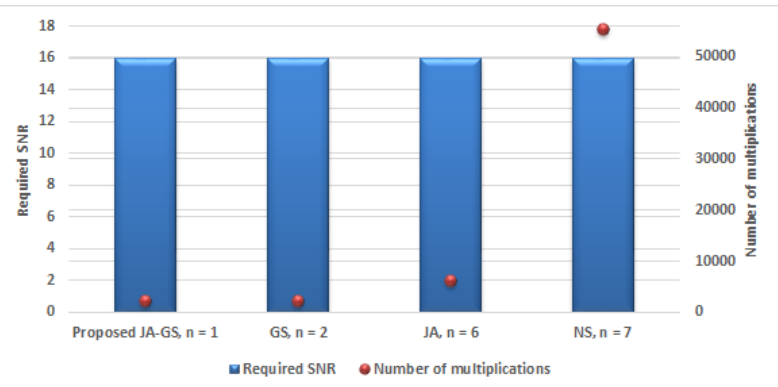

Figure 3. Performance-Complexity comparison of the NS, GS, JA, and proposed JA-GS methods to obtain BER $=10^{-4}$ in $16 \times 128$ MIMO system

Figure 3 illustrates the required SNR, number of iterations, and required multiplications to obtain $=10^{-4}$. The proposed JA-GS based detector obtains the target performance at SNR = $16 \mathrm{~dB}$ with $n=1$ while other detectors required large number of iterations to obtain the target performance. It is clear that the 
proposed detector has the lowest complexity while complexity of the NS method is highest.

\section{CONCLUSION}

In this paper, a joint JA and GS methods are utilized to propose a low complexity detector for massive MIMO UL system. The JA method has been exploited to initialize the GS method. Numerical results show that the proposed JA-GS detector achieves a performance enhancement and complexity reduction. Proposed initialization method could be applied with the SOR, the Richardson, and the CG. Moreover, proposed method could be used to initialize nonlinear based detectors such as the SD and the SIC.

\section{ACKNOWLEDGMENT}

This research is financially supported by A'Sharqiyah University Research Visits Support Fund. In addition, the research leading to these results has received funding from the Research Council (TRC) of the Sultanate of Oman under the Block Funding Program (TRC Block Funding Agreement No. BFP/RGP/ICT/18/079).

\section{REFERENCES}

[1] "Ericsson mobility report," Report, pp. 1-36, 2019.

[2] M. A. M. Albreem, "5G wireless communication systems: Vision and challenges," in 2015 International Conference on Computer, Communications, and Control Technology (I4CT), April 2015, pp. 493-497.

[3] K. Chen, C. Qi, and G. Y. Li, "Two-step codeword design for millimeter wave massive mimo systems with quantized phase shifters," IEEE Transactions on Signal Processing, vol. 68, pp. 170-180, 2020.

[4] M. A. M. Albreem, "Approximate matrix inversion methods for massive mimo detectors," in 2019 IEEE 23rd International Symposium on Consumer Technologies (ISCT), June 2019, pp. 87-92.

[5] M. Z. Chowdhury, M. T. Hossan, A. Islam, and Y. M. Jang, "A comparative survey of optical wireless technologies: Architectures and applications," IEEE Access, vol. 6, pp. 9819-9840, 2018.

[6] B. M. Lee and H. Yang, "Massive MIMO for industrial internet of things in cyber-physical systems," IEEE Trans. Ind. Informat., vol. 14, no. 6, pp. 2641-2652, June 2018.

[7] M. A. M. Albreem, A. El-Saleh, M. Isa, W. Salah, and M. Juso, "Green internet of things: an overivew," in 2017 4th IEEE International Conference on Smart Instrumentation, Measurement and Applications (ICSIMA2017), November 2017, pp. 1-6.

[8] M. Albreem, "Efficient initialization of iterative linearmassive mimo detectors using a stair matrix," Electronics Letters, October 2019.

[9] M. A. M. Albreem, A. A. El-Saleh, and M. Juntti, "On approximate matrix inversion methods for massive mimo detectors," in Proc. IEEE Wireless Commun. and Networking Conf., April 2019, pp. 1-6.

[10] M. A. Albreem, M. Juntti, and S. Shahabuddin, "Massive MIMO detection techniques: A survey," IEEE Commun. Surveys Tuts., September 2019.

[11] M. A. M. Albreem and M. F. M. Salleh, "Regularized lattice sphere decoding for block data transmission systems," Wireless Personal Communications, vol. 82, no. 3, pp. 1833-1850, Jun 2015. [Online]. Available: https://doi.org/10.1007/s11277-015-2317-2

[12] C. Chen and Y. Huang, "Lattice-reduction-aided one-bit precoding for massive mu-mimo systems," IEEE Transactions on Vehicular Technology, vol. 68, no. 7, pp. 7184-7188, July 2019.

[13] M. A. M. Albreem and M. F. M. Salleh, "Near- $\mathrm{A}_{n}$-lattice sphere decoding technique assisted optimum detection for block data transmission systems," vol. E96-B, no. 1, pp. 365-359, Jan 2013. 